\title{
POLARIZATION DECOMPOSITION AND TEMPERATURE BIAS RESOLUTION FOR SMAP PASSIVE SOIL MOISTURE RETRIEVAL USING TIME SERIES BRIGHTNESS TEMPERATURE OBSERVATIONS
}

\author{
Steven Chan ${ }^{1}$, Rajat Bindlish ${ }^{2}$, Peggy O'Neill $^{2}$, Thomas Jackson ${ }^{3}$, Andreas Colliander ${ }^{1}$, and Simon Yueh ${ }^{1}$ \\ ${ }^{1}$ NASA Jet Propulsion Laboratory, California Institute of Technology, Pasadena, CA, USA \\ ${ }^{2}$ NASA Goddard Space Flight Center, Greenbelt, MD, USA \\ ${ }^{3}$ USDA ARS Hydrology and Remote Sensing Laboratory, Beltsville, MD, USA
}

\begin{abstract}
In passive microwave remote sensing of soil moisture, the tau-omega $(\tau-\omega)$ model has often been used to provide soil moisture estimates at a spatial scale representative of the satellite footprint dimensions. For modeling simplicity, model parameters such as the single scattering albedo $(\omega)$ and vegetation opacity $(\tau)$ that go into the geophysical inversion process are often assumed to be independent of polarizations. Although this absence of polarization dependence can often be justified in special cases as in low-frequency remote sensing or under dense vegetation conditions, it is not a robust assumption in general. Additional model parameterization errors arising from this assumption are possible, leading to degradation in soil moisture estimation accuracy. In this paper, we propose a time series approach to try to resolve the polarization dependence of several $\tau$ - $\omega$ model parameters as well as the temperature bias arising from the ancillary temperature data. The Version 4 of the Soil Moisture Active Passive (SMAP) Level 1B brightness temperature time series observations were used to illustrate the mechanics of this approach, with an emphasis on a comparison between resulting satellite soil moisture retrievals and in situ data collected at several core validation sites. It was found that this time series approach resulted in significant reduction of the dry bias exhibited in the current SMAP passive soil moisture data products, while retaining the same performance in other metrics of the current baseline passive soil moisture retrieval algorithm.
\end{abstract}

Index Terms - SMAP, passive, soil moisture, time series, polarization.

\section{INTRODUCTION}

Since its launch in January 2015, the NASA Soil Moisture Active Passive (SMAP) mission has returned nearly three years of L-band $(1.41 \mathrm{GHz})$ brightness temperature observations at both horizontal and vertical polarizations. The data have enabled routine operational and near real-time production of passive soil moisture estimates with an average global revisit period of 2-3 days [1].
The current baseline passive soil moisture retrieval algorithm is a single channel algorithm that utilizes the vertically polarized brightness temperatures (SCA-V), along with ancillary data and model parameters that were designated before launch [2]. Although the baseline retrieval algorithm was found to deliver soil moisture estimates with an unbiased RMSE that exceeded the SMAP target retrieval accuracy $\left(\leq 0.040 \mathrm{~m}^{3} / \mathrm{m}^{3}\right.$ over non-frozen land surfaces with vegetation water content $\leq 5 \mathrm{~kg} / \mathrm{m}^{2}$ ), the corresponding estimates were shown to exhibit a moderate dry bias $(>0.030$ $\mathrm{m}^{3} / \mathrm{m}^{3}$ ) over a majority of core validation sites where in situ data were available for direct comparison [3][4][5]. Various causes have been proposed to explain the observed dry bias, including systematic uncertainties in the spatial aggregation of in situ data, inadequate modeling of soil moisture variability over soil depth, and uncertainties in ancillary data. In addition to these possible causes, non-optimal forward model parameters, especially those assumed to have no polarization dependence, as well as systematic bias in effective soil temperature [6] may also play a significant role in the overall level of soil moisture estimates, causing the resulting retrieval to bias in one way or the other.

In this paper, we propose a time series dual channel algorithm (TS-DCA) that attempts to address parameter optimality and surface temperature bias. A similar time series approach (MT-DCA) [7] had been proposed to solve for $\omega$ and $\tau$, assuming their independence on polarization and a constant vegetation opacity for a short period of time. In TSDCA, the inversion model is subject to the time constraints of the SMAP brightness temperature observations at horizontal and vertical polarizations. This formulation resolves not only the optimality and polarization dependence of model parameters but also the bias in surface temperature as expected by the forward model.

\section{METHODOLOGY}

In passive microwave remote sensing of soil moisture, the tau-omega $(\tau-\omega)$ model has often been used for soil moisture retrieval. A common formulation of the model for brightness temperatures observed at an angle of $\theta$ can be found in [2]: 


$$
\begin{aligned}
T_{B p}=T_{s} e_{p} \exp ( & \left.-\tau_{p} \sec \theta\right) \\
& +T_{c}\left(1-\omega_{p}\right)[1 \\
& \left.-\exp \left(-\tau_{p} \sec \theta\right)\right][1 \\
& \left.+r_{p} \exp \left(-\tau_{p} \sec \theta\right)\right]
\end{aligned}
$$

where

$$
r_{p}=r_{o p} \exp \left(-h_{p} \cos ^{2} \theta\right)
$$

In Eq. (1), the subscript $p$ refers to either horizontal or vertical polarization, $T_{s}$ is the soil effective temperature, $T_{c}$ is the vegetation temperature (often assumed to be the same as $T_{s}$ during the dawn hours for SMAP descending overpasses), $\tau_{p}$ is the nadir vegetation opacity, $\omega_{p}$ is the vegetation single scattering albedo, and $r_{p}$ is the rough-surface soil reflectivity, which is related to the smooth-surface soil reflectivity $r_{o p}$ according to Eq. 2 through the roughness coefficient $h_{p}$ in an exponential term. Eqs. 1 and 2 allow soil moisture estimates to be inverted from $T_{B p}$ (from observations), $T_{S}$ and $\tau_{p}$ (from ancillary data), as well as $h_{p}$ and $\omega_{p}$ (from lookup table) through a soil dielectric model.

The time series dual channel formulation presented in this paper accommodates polarization dependence in the roughness coefficient $\left(h_{h}\right.$ and $\left.h_{v}\right)$, single scattering albedo $\left(\omega_{h}\right.$ and $\omega_{r}$ ), and vegetation opacity ( $\tau_{h}$ and $\tau_{v}$ ), and attempts to resolve them individually. In addition, an additive bias term $(\delta)$ in $T_{s}$ is also included so as to account for potential discrepancy between the ancillary land surface model output temperature and the radiometric response as expected by the model. With a brightness temperature record with $N$ discrete time series samples at horizontal and vertical polarizations, the following inequality must be met:

$$
2 N \geq N+7
$$

before the $2 N$ brightness temperature time series observations can be used to solve for the $N$ soil moisture time series estimates and other unknown polarization-dependent parameters that are assumed to have small or no variability over a duration represented by $N$. In this presentation, a $2 \frac{1}{2}-$ year record (Apr 2015 through Nov 2017) of horizontally and vertically polarized SMAP Level 1B brightness temperature time series was formulated using the $\tau$ - $\omega$ model in a leastsquare nonlinear minimization scheme to solve for the unknown quantities $\left(m_{v}, h_{h}, h_{v}, \omega_{h}, \omega_{v}, \tau_{h}, \tau_{v}\right.$ and $\left.\delta\right)$ described above, where the soil moisture time series $m_{v}$ is a vector of dimension $N$. At each grid cell location, the roughness coefficient, single scattering albedo, and the temperature bias were assumed to be constant over time for simplicity, whereas the vegetation opacity was assumed to follow the general climatological temporal trend (thus slow temporal variability) of the normalized vegetation difference index (NDVI). A prototype of this minimization scheme was implemented in MATLAB, resulting in the extraction of a global 21/2-year record of soil moisture, vegetation opacity, and static maps of model parameters.

\section{RESULTS}

Tables 1 and 2 illustrate the resolved $T_{s}$ bias $(\delta)$ and key performance metrics (bias, correlation, and unbiased rootmean-square error [ubRMSE]) in comparisons between the TS-DCA retrieval and in situ data. The same metrics were also computed for SCA-V for comparison. Figures 1 and 2 illustrate the estimates of soil moisture $\left(m_{v}\right)$ and vegetation opacity $\left(\tau_{v}\right)$, whereas Figure 3 shows the resolved $T_{s}$ bias. Unless stated otherwise, all analyses were conducted on a 36 km EASE Grid 2.0 global projection.

Table 1: 6:00 am TS-DCA retrieval vs. in situ data

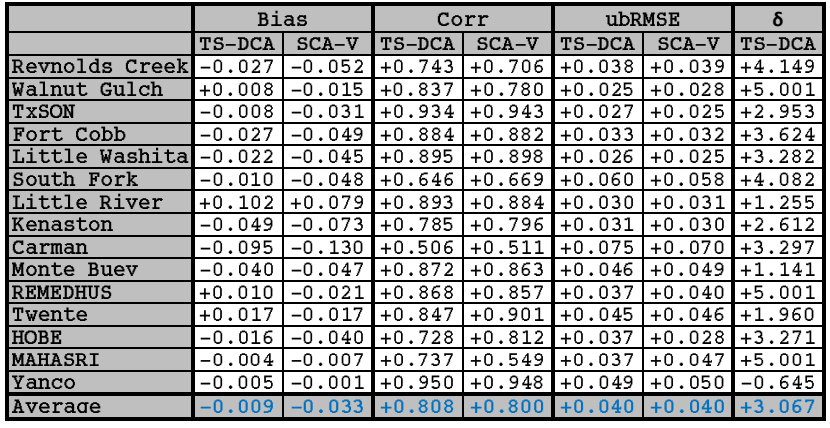

Table 2: 6:00 pm TS-DCA retrieval vs. in situ data
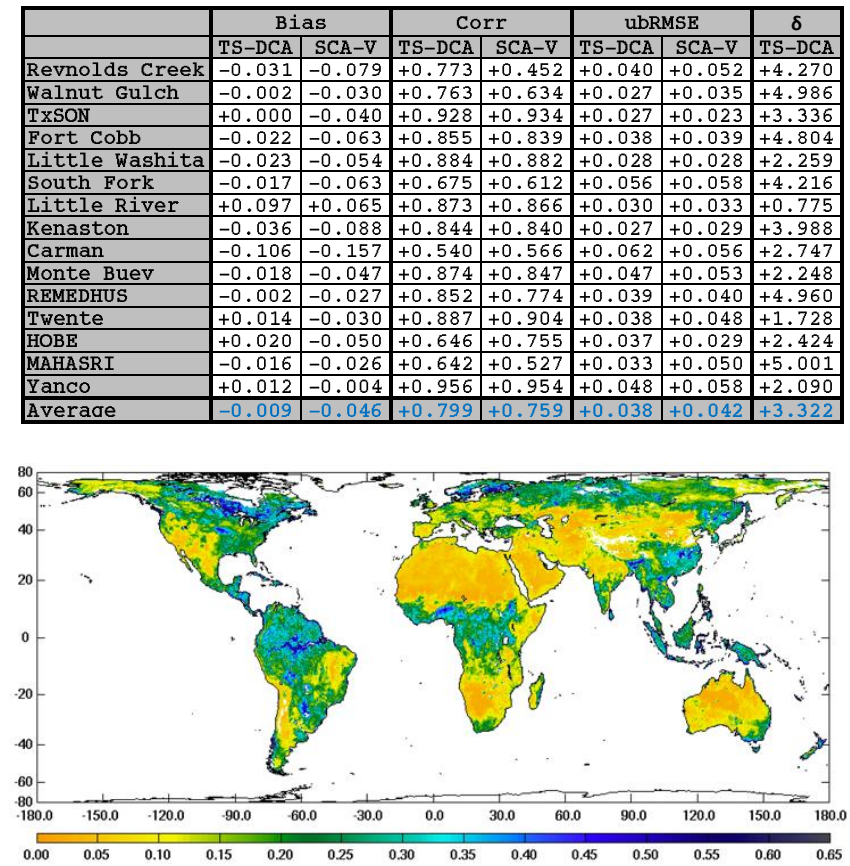

Figure 1: 6:00 am TS-DCA soil moisture estimates in $\mathrm{m}^{3} / \mathrm{m}^{3}$ between Jun 57, 2015 using a 21/2-year record of SMAP L-band brightness temperatures. 


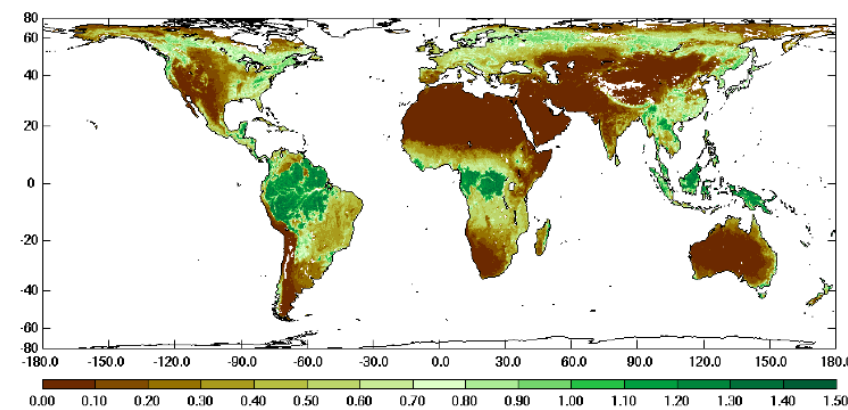

Figure 2: 6:00 am TS-DCA vertically polarized vegetation opacity estimates between Jun 5-7, 2015 using a 21/2-year record of SMAP L-band brightness temperatures.

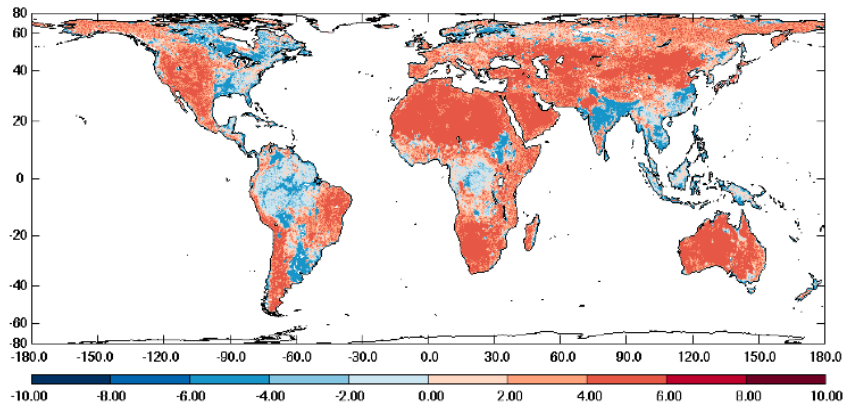

Figure 3: 6:00 am TS-DCA resolved $T_{s}$ annual mean bias using a 21/2-year record of SMAP L-band brightness temperatures.

\section{DISCUSSION}

As indicated in Tables 1 and 2, both TS-DCA and SCA-V delivered very similar performance against measured in situ data at core validation sites in terms of soil moisture retrieval accuracy. For example, the average ubRMSEs are almost identical $\left(\sim 0.040 \mathrm{~m}^{3} / \mathrm{m}^{3}\right)$ for both 6:00 am and 6:00 pm comparison; the average correlation coefficients are 0.808 vs. $0.800(6: 00 \mathrm{am})$ and 0.799 vs. $0.759(6: 00 \mathrm{pm})$. However, TS-DCA, by virtue of its more polarization-dependent model parameters and resolved bias in $T_{s}$, resulted in a far lower bias over a majority of core validation sites when compared with SCA-V: $-0.009 \mathrm{~m}^{3} / \mathrm{m}^{3}$ vs. $-0.033 \mathrm{~m}^{3} / \mathrm{m}^{3}(6: 00 \mathrm{am})$ and $-0.009 \mathrm{~m}^{3} / \mathrm{m}^{3}$ vs. $-0.046 \mathrm{~m}^{3} / \mathrm{m}^{3}(6: 00 \mathrm{pm})$. The average resolved $T_{s}$ biases according to TS-DCA are $+3.067 \mathrm{~K}(6: 00$ am) and $+3.322 \mathrm{~K}(6: 00 \mathrm{pm})$. In addition, the global distribution of soil moisture and vegetation opacity in Figs. 1 and 2 shows the expected geographical patterns, with higher soil moisture and vegetation opacity over dense forest areas and lower soil moisture and vegetation opacity over arid areas. Figure 3 suggests that the land surface model output temperature currently used in SCA-V needs warm bias correction (red areas) in more parts of the world than cold bias correction (blue areas). Global $T_{s}$ annual mean biases were found to be $+1.852 \mathrm{~K}(6: 00 \mathrm{am})$ and $+1.942 \mathrm{~K}(6: 00 \mathrm{pm})$ over land. Further independent global temperature validation analyses are needed to confirm this global pattern of $T_{s}$ bias as resolved by TS-DCA.
It is important to note that the TS-DCA comparison results in Tables 1 and 2 rely on the assumptions that (1) the $\tau$ - $\omega$ forward model is sufficiently accurate in modeling what the SMAP radiometer actually 'sees' and (2) the upscaled in situ data over core validation sites provide an accurate spatial average of soil moisture at a microwave sensing depth determined, among other factors, by surface heterogeneity, surface wetness, soil texture, vegetation water content, and soil moisture variability over depth. These assumptions are not necessarily valid over all the core validation sites considered in this work.

\section{CONCLUSION}

A time series dual channel algorithm was presented in this paper as a means to improve the dry bias exhibited in the current SMAP passive soil moisture products. Comparison with in situ data showed that the new algorithm retained the same unbiased RMSE and correlation as the current baseline retrieval algorithm (SCA-V), while significantly reducing the dry bias associated with the latter. Furthermore, the new algorithm resolves the polarization dependence of model parameters in the $\tau$ - $\omega$ model. This decomposition of polarization is expected to shed further insight into passive soil moisture retrieval algorithm development.

\section{ACKNOWLEDMENT}

This part of the work carried out at Jet Propulsion Laboratory, California Institute of Technology was performed under a contract with National Aeronautics and Space Administration.

\section{REFERENCES}

[1] Entekhabi, D., Yueh, S., O'Neill, P., Kellogg, K., 2014. SMAP Handbook - Soil Moisture Active Passive: Mapping Soil Moisture and Freeze/thaw From Space. SMAP Project, Jet Propulsion Laboratory, Pasadena, CA.

[2] O'Neill, P. E., Njoku, E. G., Jackson, T., Chan, S. K., Bindlish, R., 2015. SMAP Algorithm Theoretical Basis Document: Level 2 \& 3 Soil Moisture (Passive) Data Products. Jet Propulsion Laboratory, California Institute of Technology, Pasadena, CA (JPL D-66480). http://smap.jpl.nasa.gov/system/internal_resources/details/or iginal/316_L2 SM_P_ATBD_v7_Sep2015.pdf (accessed: December 18, 2017).

[3] Chan, S. K., R. Bindlish, P. O'Neill, E. Njoku, T. Jackson, A. Colliander, F. Chen, M. Burgin, S. Dunbar, J. Piepmeier, S. Yueh, D. Entekhabi, M. H. Cosh, T. Caldwell, J. Walker, X. Wu, A. Berg, T. Rowlandson, A. Pacheco, H. McNairn, M. Thibeault, J. Martinez-Fernandez, A. Gonzalez-Zamora, M. Seyfried, D. Bosch, P. Starks, D. Goodrich, J. Prueger, M. 
Palecki, E. E. Small, M. Zreda, J. Calvet, W. T. Crow, Y. Kerr, 2016. Assessment of the SMAP passive soil moisture product. IEEE Trans. Geosci. Remote Sens. 54 (8), 49945007.

[4] Chan, S. K., R. Bindlish, P. O'Neill, T. Jackson, E. Njoku, S. Dunbar, J. Chaubell, J. Piepmeier, S. Yueh, D. Entekhabi, A. Colliander, F. Chen, M. H. Cosh, T. Caldwell, J. Walker, A. Berg, H. McNairn, M. Thibeault, J. Martínez-Fernández, F. Uldall, M. Seyfried, D. Bosch, P. Starks, C. Holifield Collins, J. Prueger, R. van der Velde, J. Asanuma, M. Palecki, E. E. Small, M. Zreda, J. Calvet, W. T. Crow, Y. Kerr, 2017. Development and assessment of the SMAP enhanced passive soil moisture product, Remote Sensing of Environment, Volume 204, Pages 931-941. ISSN 0034-4257, https://doi.org/10.1016/j.rse.2017.08.025.

[5] Colliander, A., T. Jackson, R. Bindlish, S. K. Chan, S. Kim, M. H. Cosh, R. Dunbar, L. Dang, L. Pashaian, J. Asanuma, K. Aida, A. Berg, T. Rowlandson, D. Bosch, T. Caldwell, K. Caylor, D. Goodrich, H. al Jassar, E. LopezBaeza, J. Martinez-Fernandez, Á. Gonzalez-Zamora, S. Livingston, H. McNairn, A. Pacheco, M. Moghaddam, C. Montzka, C. Notarnicola, G. Niedrist, T. Pellarin, J. Prueger, J. Pulliainen, K. Rautiainen, J. Ramos, M. Seyfried, P. Starks, Z. Su, Y. Zeng, Y., R. van der Velde, M. Thibeault, W. Dorigo, M. Vreugdenhil, J. P. Walker, X. Wu, A. Monerris, P. E. O'Neill, D. Entekhabi, E. G. Njoku, S. Yueh, 2017. Validation of SMAP surface soil moisture products with core validation sites, Remote Sensing of Environment, Volume 191, Pages 215-231.

[6] Zeng, J., K. S. Chen, H. Bi, and Q. Chen, 2016. A Preliminary Evaluation of the SMAP Radiometer Soil Moisture Product Over United States and Europe Using Ground-Based Measurements, IEEE Transactions on Geoscience and Remote Sensing, vol. 54, no. 8, pp. 49294940, doi:10.1109/TGRS.2016.2553085.

[7] Konings, A. G., M. Piles, K. Roetzer, K. A. McColl, S. Chan, and D. Entekhabi, 2016. Vegetation optical depth and scattering albedo retrieval using time series of dual-polarized L-band radiometer observations, Remote Sensing of Environment, Volume 172, Pages 178-189. 